\title{
POSTNATAL DEVELOPMENT OF THE PHASE-LOCKED RESPONSE TO LOW FREQUENCY TONES OF AUDITORY NERVE FIBERS IN THE CAT ${ }^{1}$
}

\author{
RONALD E. KETTNER, ${ }^{2}$ JIA-ZHEN FENG ${ }^{3}$ AND JOHN F. BRUGGE \\ Department of Neurophysiology and Waisman Center on Mental Retardation and Human Development, University of \\ Wisconsin, Madison, Wisconsin 53706
}

Received December 27, 1983; Revised August 16, 1984; Accepted August 16, 1984

\begin{abstract}
The maturation of the auditory nerve's ability to encode temporal information in an acoustic signal was studied in young kittens, 7 to 23 days old, and in adult cats by measuring the degree to which auditory nerve fiber responses are synchronized (phase locked) to low frequency tones. The major findings include the following. (1) In 7- to 10-day-old kittens thresholds are high (around $100 \mathrm{~dB}$ ), and secure phase locking is observed only at frequencies below about $600 \mathrm{~Hz}$. (2) The upper frequency limit for phase locking in the adult, around $4 \mathrm{kHz}$, is reached gradually toward the end of the third posnatal week, a time when thresholds also reach their adult levels. (3) The time course of development of the phase-locked response is similar for fibers with different characteristic frequencies (CFs). (4) $\Lambda$ t all ages studied, the maximal phase synchrony at any given low frequency is generally obtained at stimulus intensities less than $20 \mathrm{~dB}$ above the rate threshold and some 10 to $30 \mathrm{~dB}$ below the saturation firing level of the fiber. (5) Time delays, estimated from phase-versusfrequency plots, were similar across ages, although they tended to be longer and more variable at lower CFs during the first 2 postnatal weeks. (6) Comparing the phase-locked response of auditory nerve fibers with that of the anteroventral cochlear nucleus neurons from a previous study suggested that the upper frequency limit for phase synchrony was reached later in the nucleus than in the nerve.
\end{abstract}

Information about the acoustic stimulus is encoded in trains of action potentials transmitted from the cochlea to the cochlear nuclei of the brainstem by fibers of the ateditory nerve. In response to low frequency sounds, below about $4 \mathrm{kHz}$, the discharge of an auditory nerve fiber is locked to a particular phase of the stimulus waveform (Rose et al., 1967; Brugge et al., 1969; Johnson, 1980). As a consequence of this, the nerve impulses within the discharge train occur at intervals that correspond to integral multiples of the period of the stimulating waveform. Such a period-time code is required by the volley theory of hearing that was put forward by Wever (1949) more than three decades ago. Low frequency time information transmitted in the auditory nerve fiber discharge is relayed relatively undistorted by phase-sensitive neurons of the anteroventral cochlear nucleus (AVCN) (Lavine, 1971; Rose et al., 1974) to

\footnotetext{
${ }^{1}$ This work was supported by National Research Service Award NS06887 to R. E. K. and by National Institute of Child Health and Human Development, Grant HDO3353. We thank Ellen Burleigh for the art work and Shirley Hunsaker for the photography. We are especially grateful to Drs. W. S. Rhode and G. L. Roth of the Department of Neurophysiology for allowing us to use some of their unpublished data on the auditory nerve of the adult cat.

${ }^{2}$ To whom correspondence should be addressed, at Department of Neurophysiology, 627 Waisman Center, University of Wisconsin, Madison, WI 53706.

${ }^{3}$ Present address: Department of Anatomy, University of Connecticut, Farmington, CT 06032.
}

binaurally sensitive cells located mainly in the superior olivary complex (Goldberg and Brown, 1969). These mechanisms for encoding and transmitting temporal information are important for sound localization (Rose et al., 1966) and probably for the perception of complex sounds such as speech (Young and Sachs, 1979; Sinex and Geisler, 1983). The experiments presented here sought to trace in auditory nerve fibers of young kittens the time course of development of the phase-locked response to low frequency tones which underlies this temporal coding mechanism.

The auditory pathway of the newborn kitten is structurally and functionally underdeveloped. Many of the structural and functional changes taking place in the external, middle, and inner ears of kittens during the first postnatal weeks (see Romand, 1983; Saunders et al., 1983) are reflected in the discharge properties of auditory nerve fibers (Carlier et al., 1975, 1979; Walsh et al., 1982; Romand, 1979, 1983). Spontaneous activity levels, rate-intensity functions, tuning curves, thresholds, and discharge patterns are significantly modified during the first few weeks after birth, and the time course of the development of these response properties runs roughly parallel to that of the peripheral auditory structures. Similar observations have been made on the functional development of the AVCN (Brugge et al., 1978, 1981). In these AVCN studies, however, it was also discovered that the low frequency phaselocking ability of neurons in this region was underdeveloped at birth and, furthermore, that maturation of this coding mechanism appeared to extend several weeks beyond the time when other functional properties of AVCN cells had fully matured. 
Thus, a question arose as to whether the underdevelopment of phase-locking ability was due to functional immaturity in the AVCN or whether these properties reflected activity in a functionally immature auditory nerve or structures peripheral to it.

\section{Materials and Methods}

Five hundred and twenty-six single auditory nerve fibers were studied in 18 kittens ranging in age from 7 to 23 days and in three young adult cats, 5 to 6 months old. Phase-locking information was obtained from 327 of these fibers. The animals were born and raised in our facilities, were in good health, and were free of ear disease. For the analysis of time delay development, presented in Figure 8 , we have included additional data on 381 adult auditory nerve fibers from an unpublished study of W. S. Rhode and G. L. Roth carried out under experimental conditions similar to those described below.

The animal preparation and sound delivery system used here were essentially the same as those used in earlier studies of the developing AVCN (Brugge et al., 1978, 1981). Kittens were anesthetized with an intramuscular injection of ketamine hydrochloride followed $0.5 \mathrm{hr}$ later by an intraperitoneal injection of sodium pentobarbital. Anesthesia was maintained with ketamine. A tracheal cannula was inserted, the left pinna was removed, and the skull was secured to a rigid head holder using dental acrylic. A small hole was made in the occipital region of the skull, and the cochlear nerve and nuclei were exposed by aspirating overlying cerebellar tissue. The auditory nerve was further exposed by gently pushing small cotton balls, wetted with saline, between the cochlear nuclei and skull wall. High impedance ( 30 to 50 megohms) micropipettes, filled with $3 \mathrm{M} \mathrm{NaCl}$, were advanced into the nerve under microscopic control using a Trent-Wells hydraulic microdrive system. The cavity around the cochlear nerve and nuclei was filled with a warm $2 \%$ agar solution to reduce brain pulsations.

Pure tones, generated by a Beyer DT- $18 \mathrm{~S}$ earphone, were delivered via a metal tube sealed into the external ear canal. The oscillator and attenuator were under computer control. Sound pressure levels (SPLs; re $0.0002 \mathrm{dyne} / \mathrm{cm}^{2}$ ) were determined using a calibrated probe tube attached to a $1 / 2$-inch Bruel \& Kjaer condenser microphone. Acoustical calibration was accomplished using a LINC computer in conjunction with a General Radio wave analyzer. For each experiment, calibration values were stored by the LINC for later use in that experiment by online data collection programs. Tones were gated on and off by an electronic switch with rise and fall times of $5 \mathrm{msec}$.

In a typical experiment, the threshold and characteristic frequency (CF) of an isolated auditory nerve fiber were determined using audiovisual cues. CF was defined as that frequency which would elicit an auditory nerve response at the lowest SPL. Thresholds were also determined at several frequencies on either side of the CF. Period histograms, which plot the distribution of spikes during one period of a tonal stimulus, were derived from 6 sec of tonal stimulation. They were displayed on-line and then stored for further analysis. A quantitative measure of phase locking, the vector strength (Goldberg and Brown, 1969), was later computed from stored histograms.

Usually it was not possible to maintain the electrode in contact with a single fiber long enough to explore in detail its entire response area. In early experiments, we concentrated on obtaining phase-locking information at many closely spaced stimulus frequencies at intensities 20 to $30 \mathrm{~dB}$ above threshold. Later, we concentrated on studying the effects of stimulus intensity on both the phase-locked response and the discharge rate.

\section{Results}

Development of threshold. The threshold for an auditory nerve fiber is related to both the fiber's age and the CF. These relationships are illustrated in Figure 1 for the 526 fibers in our sample. Threshold for a single fiber was taken as the lowest SPL that evoked a detectable increase in firing rate. The solid lines in Figure 1, $A$ to $C$, approximate the lower limit of the adult threshold range derived from data plotted in Figure $1 C$. Within the first week or so of postnatal life (Fig. 1A, open circles), thresholds often exceed $100 \mathrm{~dB}$ SPL, which is well above the adult values obtained at all but the highest frequencies studied. With advancing age, thresholds gradually decline, approaching adult values by the end of the third postnatal week. Plots of average threshold versus age for low-, medium-,
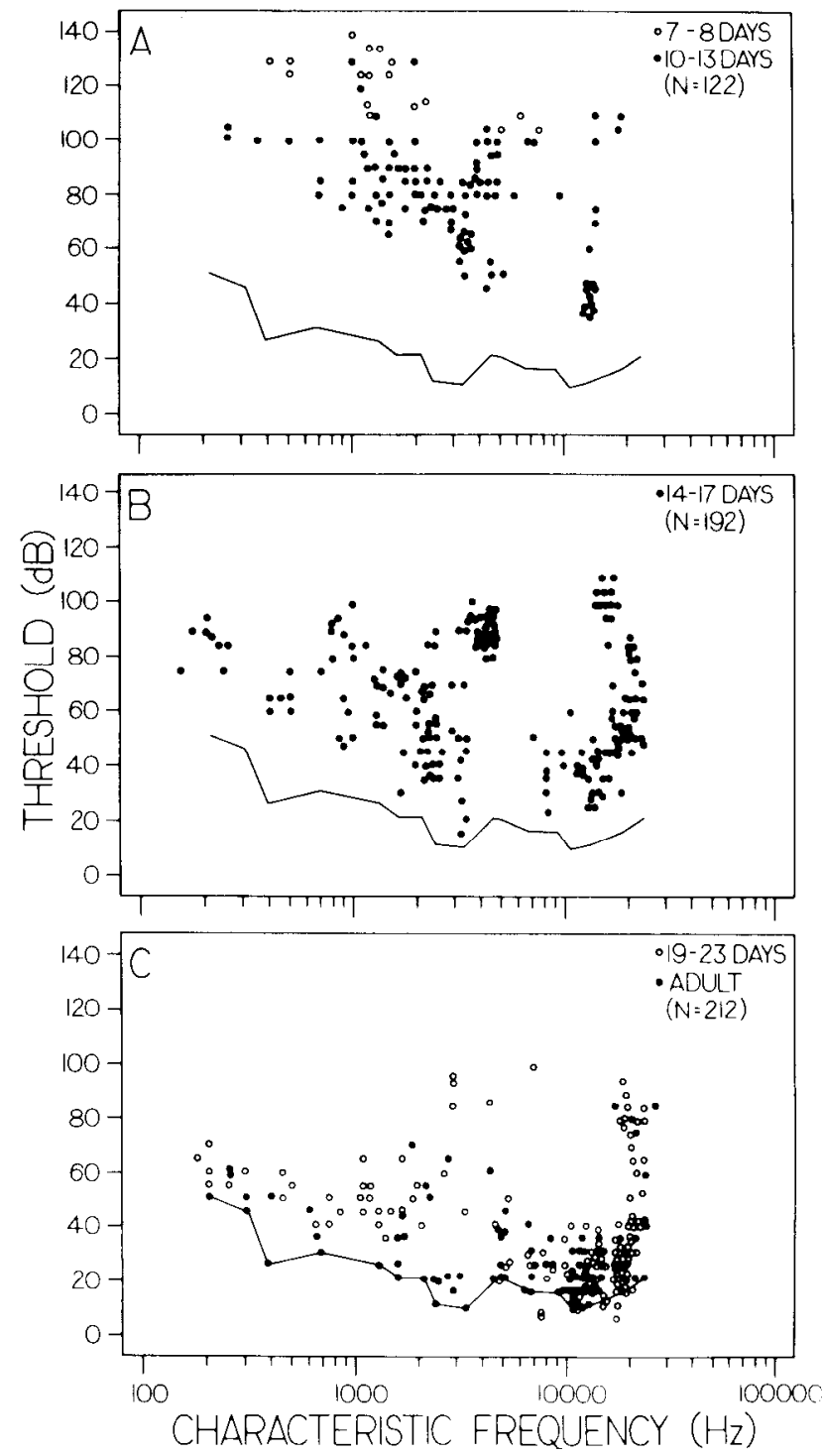

Figure 1. Threshold values at $\mathrm{CF}$ as a function of $\mathrm{CF}$ for auditory nerve fibers in young kittens and adult cats. $A$, Thresholds obtained from 7- to 8-day (O) and 10- to 13-day (-) kittens. $B$, Thresholds obtained from 14- to 17-day kittens. $C$, Thresholds obtained from 19 to 23-day kittens $(O)$ and adult cats $(-$ ). In $A$ to $C$ the solid line estimates the lowest thresholds obtained for adult cats. The number $(N)$ of auditory nerve fibers studied is displayed for each plot.

and high-CF fibers (corresponding to approximately equal distances along the basilar membrane of the adult cat) indicate that, after the first postnatal week, the time course of threshold development is independent of CF (Fig. 2).

Phase locking as a function of postnatal age. Period histograms derived from responses to four stimulus frequencies $-500,1000$, 2000 , and $3000 \mathrm{~Hz}$-are shown for both kittens and adult cats in Figure 3. Each histogram in the figure was obtained from a different auditory nerve fiber with the stimulus frequency set at, or near, the CF of that fiber and the stimulus intensity set 10 to $30 \mathrm{~dB}$ above threshold. All histograms were thus obtained from well driven responses, and most exhibited the best phase locking in a series of period histograms obtained at several intensity levels. This figure illustrates two features of the phase-locked response of auditory nerve fibers in kittens and cats. First, secure phase locking is observed at all stimulus frequencies below about $600 \mathrm{~Hz}$ in adult cats and in kittens as young as 7 days of age. Second, as the stimulus frequency is raised, the degree of phase synchrony falls off more rapidly in 


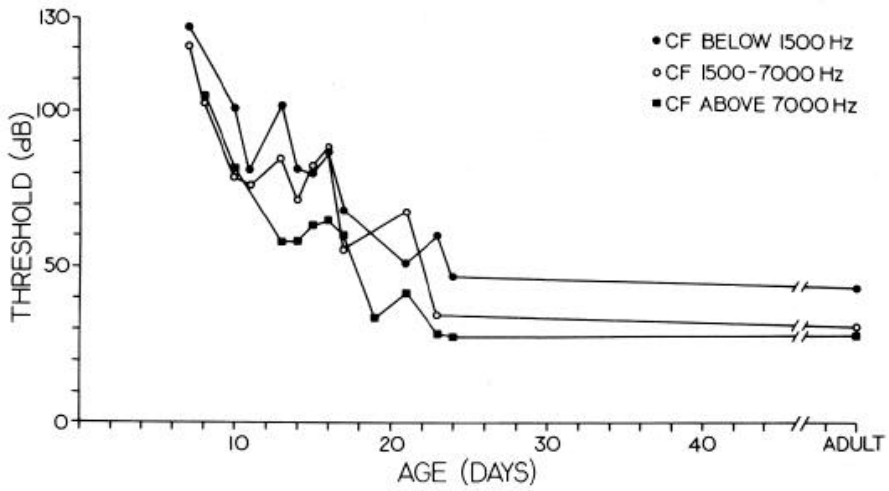

Figure 2. Average threshold values for auditory nerve fibers plotted as a function of age for three ranges of CF: CFs below $1500 \mathrm{~Hz}(\bullet)$, CFs between 1500 and $7000 \mathrm{~Hz}(\mathrm{O})$, and CFs above $7000 \mathrm{~Hz}$ (匹).

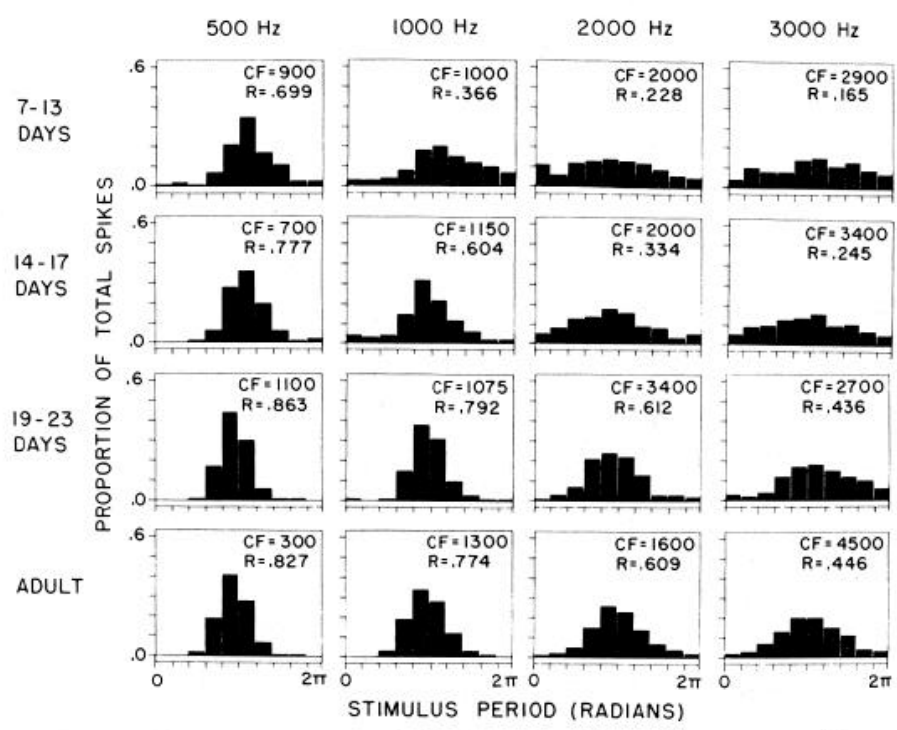

Figure 3. Representative period histograms for auditory nerve fibers stimulated at $500,1000,2000$, and $3000 \mathrm{~Hz}$ at different postnatal ages. The CF of the fiber studied and the vector strength $(R)$ of the response are displayed above each histogram. Each histogram consists of 10 equal bins which represent the proportion of spikes falling in each of 10 equal parts of the stimulus cycle. The first bin labeled 0 corresponds to the initial tenth of the stimulus cycle, and the final bin labeled $2 \pi$ corresponds to the final tenth of the stimulus cycle.

young kittens that it does in older kittens and adult cats. Phase locking becomes noticeably reduced by $1000 \mathrm{~Hz}$ in kittens 7 to 13 days of age and by $2000 \mathrm{~Hz}$ in kittens 14 to 17 days of age, but is as strong at $3000 \mathrm{~Hz}$ for kittens 19 to 23 days of age as it is for adult cats.

To compare the phase-locked activity at different ages and under different stimulus conditions for all fibers in our sample, we computed for each period histogram the vector strength, which is a measure of the degree to which the spikes are locked to the stimulus cycle (Goldberg and Brown, 1969). The vector strength ranges from 0 to 1 ; a value of 0 is associated with a flat period histogram indicating no relationship of spike firing to a particular phase of the stimulus, whereas a value near 1 is associated with a strongly peaked, unimodal period histogram indicating that impulses are tightly grouped around a particular phase angle of the stimulus waveform. These values appear on the histograms of Figure 3.

The nine plots of vector strength as a function of stimulus frequency displayed in Figure 4 reveal more clearly the relationships between discharge synchrony and postnatal age, and they take into account as well the CF of the fiber. Here, vector strength is plotted against stimulus frequency for kittens 7 to 13,14 to 17 , and 19 to 23 days of age. Again, stimulus strength was held 10 to $30 \mathrm{~dB}$ above the rate threshold. The solid lines in Figure 1, $A$ to $I$, are a plot of the average vector strength determined from the discharges of adult auditory nerve fibers with CFs in the range indicated. Data from each age group have been divided into three CF ranges, below $1500 \mathrm{~Hz}, 1500$ to $7000 \mathrm{~Hz}$, and above $7000 \mathrm{~Hz}$, which correspond to approximately equal distances along the basilar membrane in the adult cat (Greenwood, 1961; Liberman, 1982). These CF-versus-place assignments remain tentative, of course, for the function relating CF to position on the basilar membrane has yet to be determined for the developing kitten cochlea.

In general, there was a gradual development in phase-locking strength during the first 3 postnatal weeks which was similar for all three ranges of CF. The degree of phase locking in auditory nerve fibers of the youngest kittens in our series tended to fall well below the adult base line values except at very low stimulus frequencies (Fig. $4, A$ to $C$ ). With advancing age, the synchronized response was recorded at progressively higher stimulus frequencies (Fig. $4, D$ to $I$ ), and by the end of the third postnatal week both the magnitude of the phaselocked response and the frequency range over which it was recorded were virtually indistinguishable from those values derived from the adult.

For any one animal, or for different animals of the same age, a certain amount of variability in the degree of phase locking was observed at any given stimulus frequency, even among fibers with the same or similar CFs. In some cases, this was no doubt due to the fact that the maximal synchrony at any given frequency was not obtained. As described later, the degree to which spikes lock to the stimulus cycle depends on the stimulus intensity at all ages. This variability is reduced when the maximal vector strength at $\mathrm{CF}$ is plotted as a function of postnatal age (Fig. 5). These data were drawn from experiments in which phase synchrony was studied as a function of stimulus intensity (see Fig. 6). For the 91 fibers for which such data are available, there is a clear and gradual improvement in phase locking between 7 and 23 days of age, at which time phase locking is indistinguishable from that recorded in the adult cat. In other cases, variability may have resulted from differences in the state of development of the cochlea and auditory nerve of animals of the same age. For example, in one 17-day-old kitten, phase locking was considerably more secure than it was in three other kittens of the same age but from different litters. Data from this kitten fall well within the distribution of points obtained from older kittens and adult cats (Fig. 4,D and $E$, open circles). This 17-day-old kitten was the youngest in the series to show mature phase locking over the range of frequencies studied.

There appeared to be little, if any, systematic difference in the phase-locked response among low-, mid-, and high-CF fibers within each age group. Those apparent differences seen in the overall distribution of vector strength values as one goes from low- to high-CF plots can generally be attributed to sampling bias. For instance, high-CF fibers were rarely isolated in the youngest kittens. Therefore, the data plotted in the high CF graph (Fig. $4 C$ ) were all obtained at 13 days of age, whereas those plotted for the low- and mid-CFs (Fig. 4, $A$ and $B$ ) were obtained from kittens in the age range of 7 to 13 days. Within any age group, the differences in phase locking for fibers of different CF ranges appear to result from the development of phase locking across days rather than from a base-to-apex developmental gradient within the cochlea. The paucity of highCF fibers in very young kittens may result from the inability to excite such fibers acoustically. Thresholds are frequently well above $100 \mathrm{~dB}$ during the first postnatal week, and our 


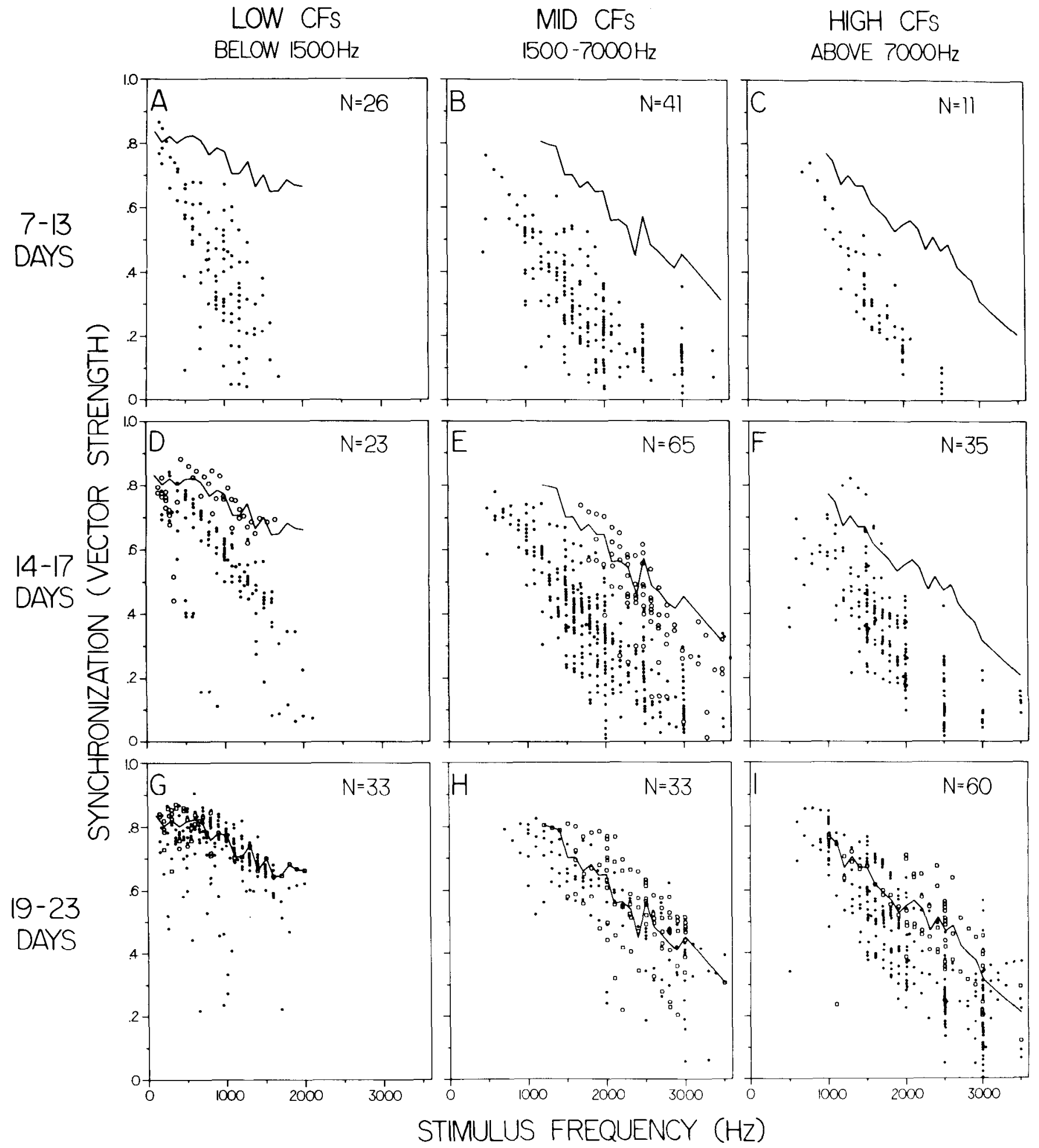

Figure 4. Vector strength plotted as a function of stimulus frequency for three ranges of CF and three postnatal age groups. In each plot the solid line represents the average adult vector strength for the appropriate range of CF. These adult curves are based upon adult values which are displayed in $G, H$, and $I$ as open squares; values obtained from 19- to 23-day animals are displayed as solid circles. The open circles in $D$ and $E$ represent values obtained from a single 17-day-old kitten which showed mature phase synchrony over the range of frequencies studied; values obtained from three other 17-day kittens and from 14- to 16-day kittens are displayed as solid circles in $A$ to $F$. The number ( $N$ ) of auditory nerve fibers studied is displayed at the top of each plot. Stimulus intensities were 10 to $30 \mathrm{~dB}$ above a fiber's rate threshold.

stimulating system was limited to levels around $100 \mathrm{~dB}$ for frequencies above $7000 \mathrm{~Hz}$.

Another example of a sampling bias which favored a particular range of CFs is seen in the data obtained from kittens 14 to 17 days old. For this group, phase locking appears more secure in some fibers having low- and mid-range CFs than in those fibers with high CFs. A closer examination indicates that the high values of phase locking observed for low-and midrange CFs in this age group are generally from that single 17 day-old kitten mentioned previously. When the open circles on 


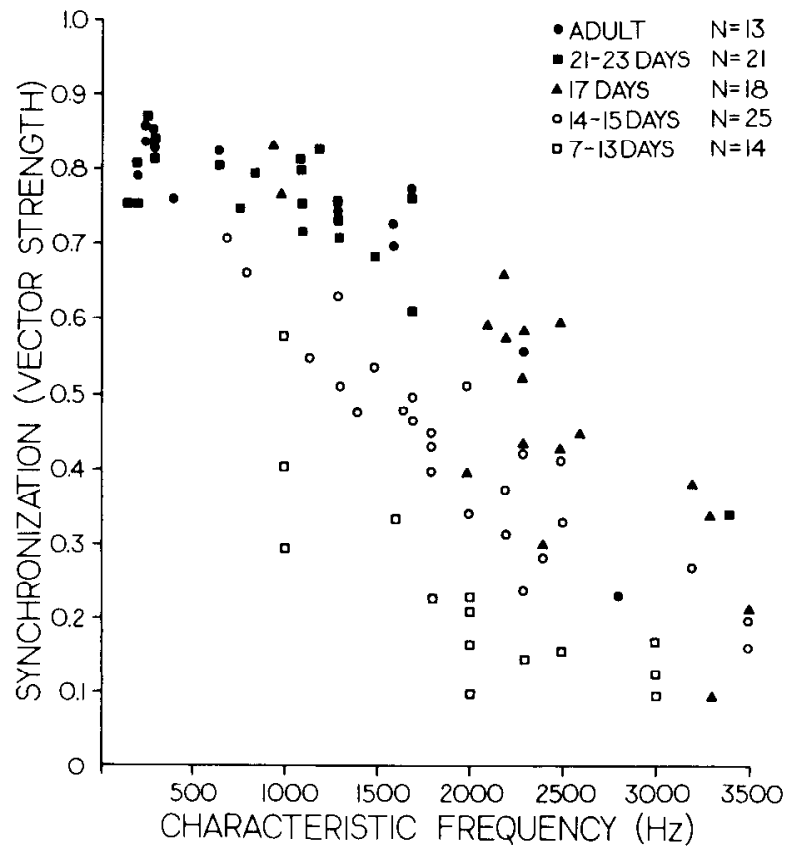

Figure 5. Maximal vector strength at $\mathrm{CF}$ as a function of $\mathrm{CF}$ for auditory nerve fibers from several postnatal age groups: adults (†), 21 to 23 days $(\boldsymbol{\square}), 17$ days $(\mathbf{A}), 14$ to 15 days $(0)$, and 7 to 13 days $(\square)$. Data at 17 days were obtained from the single 17 -day kitten which showed mature synchrony at frequencies up to $3 \mathrm{kHz}$.

this plot (Fig. 4) are ignored, the results obtained from the different $\mathrm{CF}$ ranges are nearly identical.

There were some differences in the phase-locked responses obtained from fibers with different CFs for a given age group, but these differences were generally small. In the graphs displaying low-CF results (Fig. $4, A, D$, and $G$ ), there are several data points well below the general distribution of vector strengths. These low values are less commonly encountered for mid-and high-CF fibers, and there was no obvious sampling bias in the collection of these data. Furthermore, these responses were obtained well within the response areas of the fibers with which they were associated.

Influence of stimulus intensity on the phase-locked response. As a rule, at all ages phase locking becomes more pronounced as the intensity of the stimulus is increased above threshold. Synchrony usually reaches a maximum at intensity levels less than $30 \mathrm{~dB}$ above the fiber's threshold at the frequency being used. At higher SPLs, the degree of phase locking generally levels off or decreases slightly. This relationship is illustrated in Figure 6 for a fiber from a 14-day-old kitten and one from an adult cat. Vector strength and discharge rate are plotted as a function of stimulus intensity at CF and at one frequency on either side of CF. In the kitten, the vector strength increased sharply as intensity was raised at each frequency, reached a maximal value 10 to $30 \mathrm{~dB}$ above threshold, and decreased slightly as stimulus intensity was increased even further. The curves from the adult cat leveled off around 20 to $30 \mathrm{~dB}$ above threshold at vector strengths considerably above those in the kitten, as described previously. Discharge rates in both kitten and cat reached saturation firing levels some 10 to $30 \mathrm{~dB}$ above the intensity at which phase locking reaches a maximum. This finding, that phase locking reached a maximum at stimulus intensities below that at which discharge rate saturates, is common among the fibers of our sample for both kittens and cats and agrees with earlier observations of Johnson (1980).

In those fibers showing spontaneous activity, phase locking was often demonstrable at SPLs below those needed to raise the firing rate, a finding that is common in the adult animal
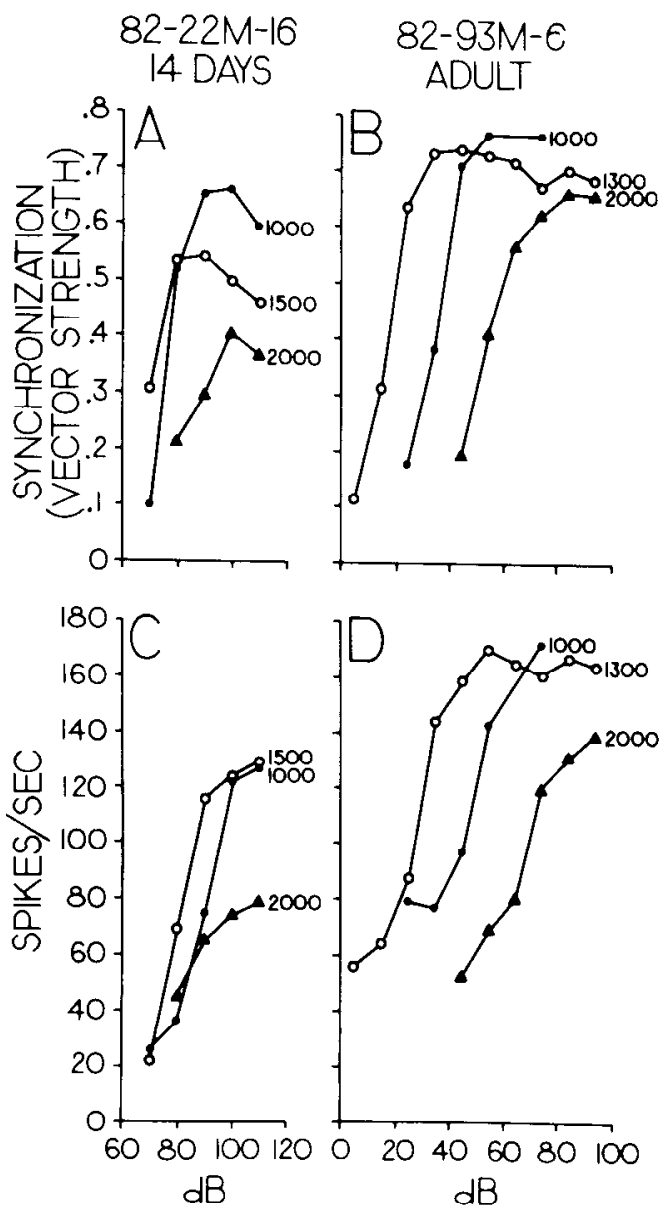

Figure 6. Vector strength $(A$ and $B)$ and firing rate $(C$ and $D)$ as functions of stimulus intensity at $\mathrm{CF}$ and at one frequency on either side of CF for two auditory nerve fibers, one from a 14-day-old kitten and one from an adult cat.

(Rose et al., 1967, 1975; Johnson, 1980). This phenomenon was studied in 265 rate-versus-intensity curves from 142 fibers in kittens 7 to 23 days of age. Phase locking was maximal at, or below, the rate threshold for $31 \%$ of the fibers studied. Maximal synchrony was reached at intensities 1 to $10 \mathrm{~dB}$ above rate threshold for $46 \%$ of the fibers, 11 to $20 \mathrm{~dB}$ above threshold for $17 \%$, and at SPLs greater than $20 \mathrm{~dB}$ above threshold for the remaining $6 \%$ of the fibers. The proportions were not significantly different for different age groups. This analysis also indicates that the vector strength values used in constructing Figure 4, which were obtained at intensities between 10 and $30 \mathrm{~dB}$ above rate threshold, reflect accurately the development of phase locking.

Development of time delays as estimated from phase-versusfrequency plots. When stimulus frequency is changed at a constant SPL, over the range of frequencies where phase locking is evident, there is a concomitant shift in the average phase of the discharge. The data points relating changes in stimulus frequency to the cumulative phase shift were fit by a straight line $(r>0.97)$. Figure 7 shows phase-versus-frequency plots for five auditory nerve fibers having CFs between 350 and 14,100 $\mathrm{Hz}$ in kittens 10 to 13 days of age. The slope of the line is related to the $\mathrm{CF}$ of the fiber; there is a progressive decrease in slope with increasing CF. Similar results were obtained at other ages.

The slopes of these and other phase-versus-frequency plots were used to estimate time delays which included acoustic and middle ear delays, travel time along the basilar membrane, and neural delays between the cochlea and the auditory nerve 


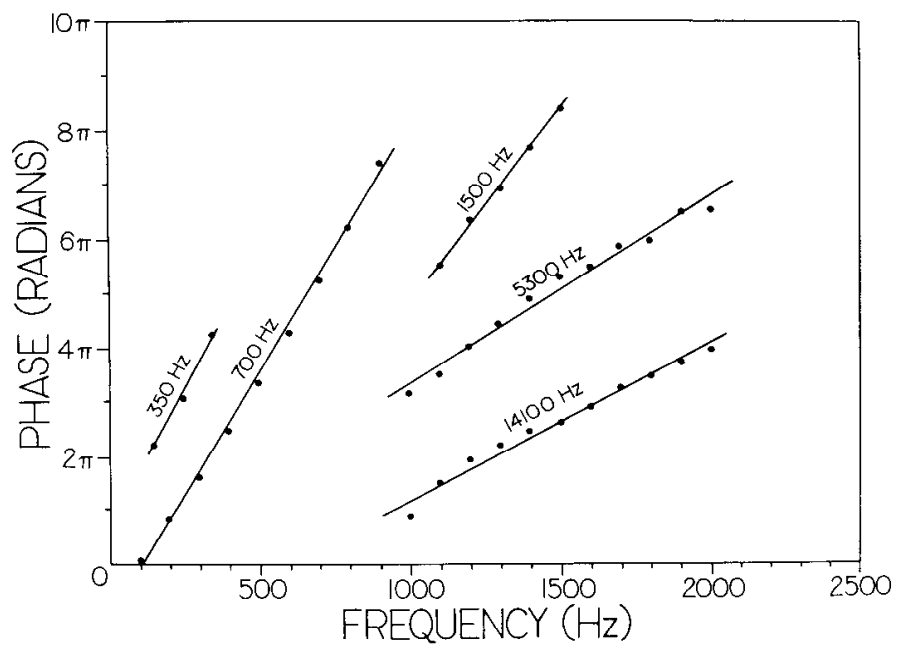

Figure 7. Cumulative phase plotted as a function of stimulus frequency for five auditory nerve fibers in kittens between 10 and 13 days of age. Average phase angle was determined from the same period histograms from which vector strength was calculated. The CF of the fiber is given above each curve. Data points are fitted by linear regression lines $(r>0.97)$. Some curves have been shifted along the phase axis to avoid crowding.

recording site. This time delay in seconds is equal to: $\Delta \theta / 2 \pi \Delta f$, where $\Delta \theta$ is the cumulative phase shift in radians observed for a change in frequency of $\Delta f$ measured in hertz (see Anderson et al., 1971, for a more detailed discussion of this analysis).

Time delays derived in this fashion are plotted in Figure 8 as a function of CF for 152 fibers in kittens younger than 24 days of age and for 433 fibers in adult cats. Data points from kittens 19 to 23 days old (Fig. 8C) were generally clustered around the average base line curve (Fig. 8 , solid line) computed from adult values (Fig. $8 D$ ). For kittens 17 days of age and younger (Fig. 8, $A$ and $B$ ), estimates of delays are close to those of the adult especially for fibers with CFs above $3 \mathrm{kHz}$; for fibers with intermediate $\mathrm{CFs}$, between 1 and $3 \mathrm{kHz}$, delays tended to be somewhat longer and tended to vary more between fibers as compared to the adult.

Comparison of the phase-locked response in the auditory nerve and AVCN. The phase-locking strength of AVCN neurons and auditory nerve fibers in kittens of various ages during the first weeks of postnatal life are compared in Figure 9. Here, as in Figure 4, vector strength is plotted as a function of stimulus frequency at SPLs 10 to $30 \mathrm{~dB}$ above the rate threshold of each fiber. Solid circles in Figure 9 represent auditory nerve results and open circles are derived from an earlier study of the AVCN (Brugge et al., 1978) carried out under the same experimental conditions as the auditory nerve work. During the first postnatal week, phase locking in both nerve and nucleus is high at very low frequencies, but drops off rapidly as frequency is raised; at $1000 \mathrm{~Hz}$, for instance, there is little sign of timelocked spikes. At this time, the distribution of points representing the auditory nerve is contained in that representing the AVCN, although, on the average, the discharges of auditory nerve fibers appear to be more securely phase locked than those of AVCN neurons at any given frequency. During the second postnatal week there is little overlap in the distributions of data points from nerve and nucleus; the phase locking at any given frequency is, as a rule, more secure for auditory nerve fibers than it is for AVCN neurons. The functional separation of nerve and nucleus remains pronounced in the third postnatal week, a time when phase locking is reaching maturity in the auditory nerve. At this point in time there appear to be two populations of AVCN neurons emerging. Our interpretation has been that one group represents those cells which have an

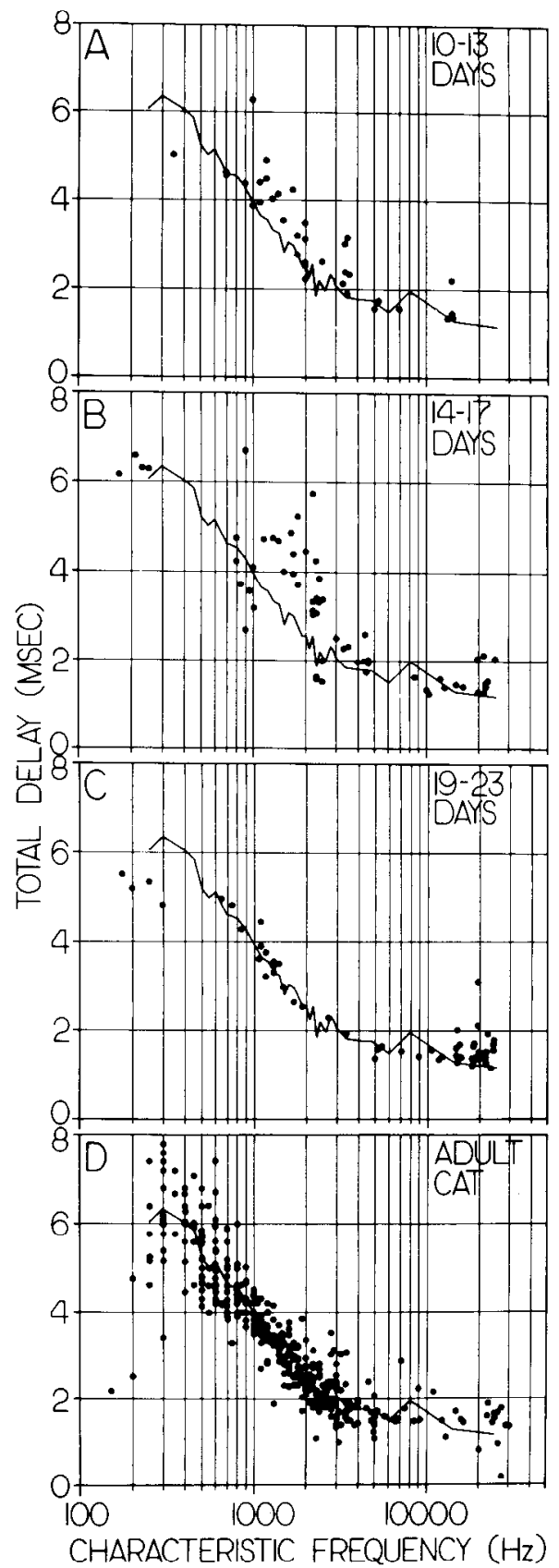

Figure 8. Total delay times, computed from phase-versus-frequency plots (see the text), as a function of CF for auditory nerve fibers from four age groups: $A, 10$ to 13 days, $N=35 ; B, 14$ lo 17 days, $N=61$; , 19 to 23 days, $N=54$; and $D$, adults, $N=433$. In $A$ to $C$ the solid line represents the average adult values for delay computed from data points plotted in $D$. In $D, 381$ fibers are from the unpublished work of W. S. Rhode and G. L. Roth.

upper frequency limit for phase locking around 600 to $1000 \mathrm{~Hz}$ in the adult cat, whereas the second group corresponds to cells whose phase-locked behavior is destined to be identical to that of auditory nerve fibers. Apparently, that point has not been reached at 3 weeks by the more phase-locked population for, compared to the auditory nerve, it is rare to find by the end of the third postnatal week an AVCN neuron that is well phase locked above about $2 \mathrm{kHz}$. Even at 8 weeks postpartum and later, when the phase-sensitive neurons in the AVCN have clearly emerged, the timing of the discharges of auditory nerve fibers is, in general, more secure than that of the phase-sensitive AVCN cells. 

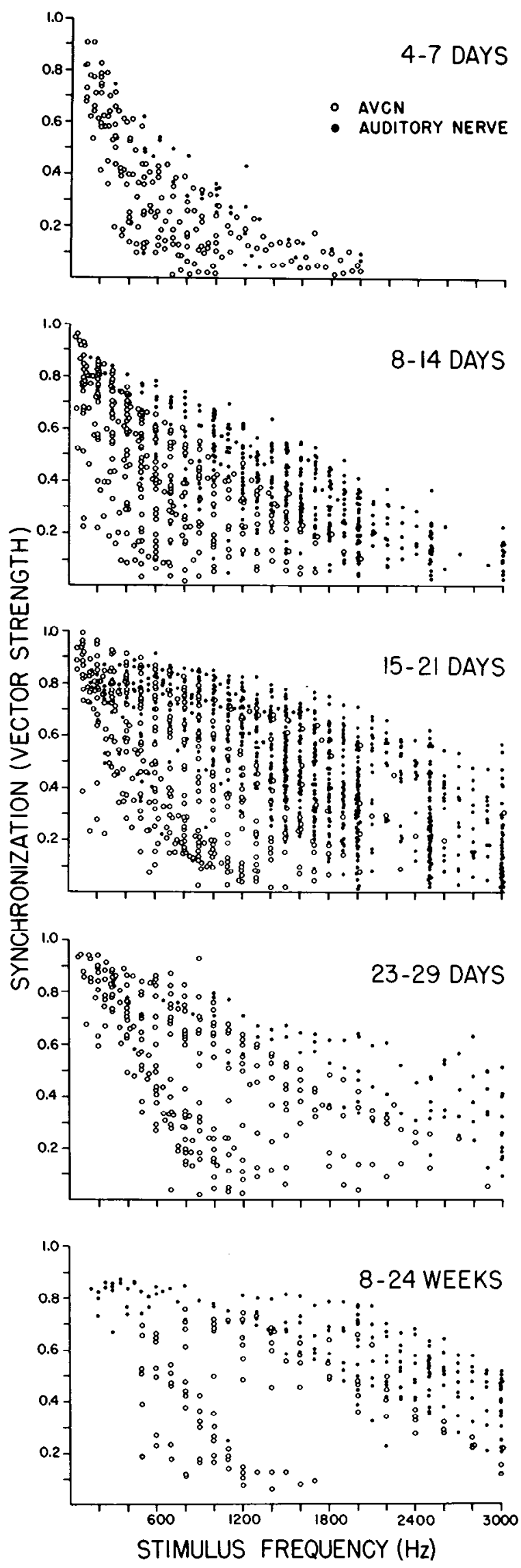

Figure 9. Plots of vector strength versus stimulus frequency for neurons of the AVCN $(O)$ and fibers of the auditory nerve $(0)$ for different age groups of kittens. The AVCN data are from a previous

\section{Discussion}

Phase-locked responses recorded from the auditory nerve of young kittens 7 to 10 days of age are immature except at stimulus frequencies below about $600 \mathrm{~Hz}$. Phase locking develops gradually over the next week or two until mature responses are obtained toward the end of the third postnatal week. At this time, rate thresholds and time delays also approach adult values.

Major factors that limit phase locking in young kittens could lie within the cochlea. The organ of Corti continues to develop for several weeks after birth, but by the end of the first postnatal week in the kitten, the gross receptor potential (cochlear microphonic, CM) recorded at the round window is evoked over nearly the full range of frequencies to which the adult cat is sensitive, although the amplitude of this potential is still relatively low as compared to the adult (Romand, 1971). Assuming that the CM in the newborn is derived from the same sources as in the adult, then at least some cochlear hair cells are responding to tones in a time-locked way at frequencies above $600 \mathrm{~Hz}$ at a time when auditory nerve phase locking at these frequencies is weak or nonexistent. This would seem to indicate that the basilar membrane and other peripheral structures are transducing signals normally over a wide range of stimulus frequencies. From intracellular recordings obtained from inner hair cells, Russell and Sellick (1978) have shown that the amplitude of the AC component of the tone-evoked, intracellular potential is attenuated with respect to the $\mathrm{DC}$ component as frequency rises. They suggest that this attenuation is due to the effect of membrane capacitance and resistance on the AC response and that this mechanism may contribute to the disappearance of phase synchrony around $5 \mathrm{kHz}$ in the adult auditory nerve fiber. Assuming that the mechanisms involving passive electrical properties of hair cells which govern the neural excitation of auditory nerve fibers are the same in cat and kitten, then the changes in auditory nerve phase locking associated with advancing age may reflect changes in the membrane properties of the developing hair cells to which these fibers are attached.

The relevant developmental changes may also take place central to the receptors, at the synapse between the hair cell and auditory nerve fiber. At birth, the inner hair cells of the cat cochlea and the afferent endings with which they make contact are in place (Ginzberg and Morest, 1983) but are immature in structure (Pujol and Marty, 1970; Pujol et al., 1978). During the next few weeks, the afferent synapses become further differentiated in that the number of vesicles increases and synaptic ribbons are encountered more frequently (see also Sobkowicz et al., 1982). According to Pujol et al (1978), the cat cochlea looks mature from a synaptic point of view by the end of the third postnatal week. This time course of development of afferent synapses on inner hair cells, from which we assume that most if not all of our auditory nerve recordings were derived, is highly correlated with that of threshold and the phase-locking ability of auditory nerve fibers. It is also close to the time course of development of other functional properties of the auditory nerve (Romand, 1983) and the cochlear nuclei (Brugge, 1983), with the exception of AVCN phase locking which appears to reach maturity several weeks later.

Finally, developmental changes in auditory nerve response properties could result from immaturities associated with the auditory nerve itself. Spiral ganglion cells, including their central processes, are relatively small and possess little myelin by the end of the first postnatal week (Romand et al., 1976, 1980;

study of Brugge et al (1978). The numbers of units recorded from the auditory nerve and the AVCN were, respectively, 14 and 14 at 4 to 7 days; 97 and 27 at 8 to 14 days; 161 and 35 at 15 to 21 days; 21 and 25 at 23 to 29 days; 37 and 13 at 8 to 24 weeks. 
Romand and Romand, 1982). If properties of auditory nerve fibers are like those of other peripheral nerves, then the conduction velocity and the length of the refractory period associated with auditory nerve fibers are most certainly influenced by axonal size and myelination. If lower conduction velocity in an auditory nerve fiber is shared by all action potentials in a discharge, then this factor alone should not disrupt synchrony. The absolute refractory period is not the factor limiting phase locking in the adult auditory nerve at moderate discharge rates (Rose et al., 1967; Johnson and Swami, 1983). Thus, a lengthening of the absolute refractory period alone would tend to reduce the probability that certain interspike intervals would occur but would not necessarily reduce the precision of firing on the stimulus cycle. Ferrington and Rowe $(1980,1982)$ ohserved that the ability of rapidly adapting cutaneous afferent fibers to encode the frequency of a vibratory stimulus applied to the footpad in the temporal rhythm of the spike discharge is demonstrably poorer in kittens than in adult cats. They attribute this functional underdevelopment more to the structurally immature receptor than to the relatively long refractory period or low conduction velocity associated with these young fibers. Whatever the mechanisms involved, if a longer refractory period and/or lower conduction velocity leads to greater variability in the time at which a spike occurs, then the upper frequency limit for phase locking would be reduced.

Gaumond et al. (1982) have indicated that under conditions of high discharge rate, such as occurs during a brief period following the onset of a suprathreshold tone burst, there are potentially two time delays that can add to the true time of occurrence of a spike, and both of these can influence phase synchrony. One may occur because spikes in a robust discharge are reduced in amplitude when they fall during the afterpotential of a preceding spike; consequently the time of occurrence of spikes in such a train are determined at different points on the spike waveforms by the fixed threshold detection method employed in these and most other experiments of this kind. In the auditory nerve of the kitten, the maximal discharge rate is low as compared to the adult, and although there were in our experiments some fluctuations in spike amplitudes during the $6 \mathrm{sec}$ of tonal stimulation used, these were not related in any obvious way to the age of the animal. Gaumond et al. (1982) suggested that the second source of this added delay is physiological and may relate to conduction time within an auditory nerve fiber. This delay is a function of the elapsed time since the occurrence of the previous spike and may result from conduction blockage related to the relative refractory period of auditory nerve fibers. If this source of added delay were greatly increased and more variable in immature axons, it could explain some of the developmental changes in phase locking which are observed.

Anderson (1973) hypothesized that the frequency-dependent decrease in phase synchrony in the adult auditory nerve response is due to a "jitter" of some 50 to $110 \mu \mathrm{sec}$ in the arrival time of axonal depolarization at the recording site. The presence of a constant amount of neural "jitter" can account for the decline in phase locking with increasing stimulus frequency, because a constant "jitter" would become more and more disruptive to the phase-locked response as the stimulus period became shorter and shorter. Similarly, a decline in the amount of "jitter" with increasing postnatal age would predict an increase in phase locking at all stimulus frequencies, but this increase would be more pronounced at higher stimulus frequencies.

As in other mammals, morphological changes in the kitten cochlea appear to follow a roughly base-to-apex developmental gradient (Romand, 1983). This would suggest that during early development the base of the cochlea may be functionally more mature than the apex. In the adult, the CF of an auditory nerve fiber corresponds to its innervation site along the basilar membrane (Liberman, 1982), with high-CF fibers arising from the base of the cochlea and low-CF fibers coming from the apex. If a similar CF-place relationship holds for young kittens, then high-CF fibers might be expected to show the first signs of functional maturity. In our results, there were no age-related changes in phase synchrony or minimum threshold that could be systematically related to the fiber $\mathrm{CF}$. On the other hand, during the first 2 postnatal weeks, delays associated with lowCF fibers tended to be somewhat longer and more variable than those recorded at later times. In young kittens these relatively long delays could involve several factors including travel time along the basilar membrane, delay at the hair cell-auditory nerve synapse, and impulse conduction time. There is evidence that, in the chick, the functional length of the cochlear partition is compressed toward the relatively mature cochlear base (Lippe and Rubel, 1983; Rubel and Ryals, 1983). If such a functional shortening of the cochlea occurs in young kittens (see Romand, 1983), then active fibers would be derived mainly, if not exclusively, from the more basal part of the cochlea, with the result that delays would tend to be shorter, rather than longer, as compared to the adult.

This does not imply that a functional developmental gradient in the kitten cochlea does not exist. According to Pujol and Marty (1970), the structural development of the apical region of the kitten cochlea lags that of the base by about 3 days. Using the date of birth as our point of reference, there is some variability in the maturity of kittens at birth as evidenced by the range of body and brain weights recorded at that time and by the fact that, at any postnatal day during the first month of life, there is scatter in response measures from both the auditory nerve and cochlear nuclei (Brugge, 1983). 'I'hus, developmental gradients in phase locking strength and average threshold may have simply been masked by the fact that on any given day these kittens were at different developmental stages.

Since the development of both the phase-locked input to and the phase-locked output from AVCN neurons were compared, any differences between the two must be the result of an underdevelopment of the AVCN neurons, the auditory nerve terminals that contact them, the degree of synchrony of the afferent input, or some combination of the three. This comparison between nerve and nucleus assumes, of course, that the sampling bias of AVCN neurons is independent of the animal's age. Moore (1981) has reported that the development of the compound action potential (AP) of the auditory nerve is not completed until some 9 weeks after birth. Since single auditory nerve fibers appear functionally mature by the end of 3 weeks of life, the relatively late AP development may result from, as Moore (1981) points out, a relatively low cross-fiber synchrony which could result from the structural underdevelopment of auditory nerve fibers (Romand et al., 1976). Phase-sensitive AVCN neurons in the adult cat presumably require highly synchronized phase-locked input from only a few auditory nerve fibers. Thus, even if the individual auditory nerve fibers converging on the AVCN neuron transmit securely phaselocked spikes, any small differences in transmission properties from one afferent fiber to the next could desynchronize the net output of that AVCN neuron. Ferrington and Rowe (1980) interpreted their findings on the development of temporal coding in second-order neurons of the cuneate nucleus in a similar way.

The thresholds of auditory nerve fibers and AVCN neurons follow the same developmental time course as that of phase locking in the auditory nerve. These findings, coupled with the relatively late development of phase synchrony in the AVCN, suggest that the kitten's ability to detect an acoustic stimulus might develop earlier than its ability to discriminate sounds 
using temporal cues. This latter ability may be, after the third week of life, limited less by the conditions of the auditory nerve and structures peripheral to it than by the state of functional development of the central auditory pathways.

\section{References}

Anderson, D. J. (1973) Quantitative model for the effects of stimulus frequency upon synchronization of auditory nerve discharges. J. Acoust. Soc. Am. 54: 361-364.

Anderson, D. J., J. E. Rose, J. E. Hind, and J. F. Brugge (1971) Temporal position of discharges in single auditory nerve fibers within the cycle of a sine-wave stimulus: Frequency and intensity effects. J. Acoust. Soc. Am. 49: 1131-1139.

Brugge, J. F. (1983) Development of the lower brainstem auditory nuclei. In Development of Auditory and Vestibular Systems, R. Romand, ed., pp. 89-120, Academic Press, Inc., New York

Brugge, J. F., D. J. Anderson, J. E. Hind, and J. E. Rose (1969) Time structure of discharges in single auditory nerve fibers of the squirrel monkey in response to complex periodic sounds. J. Neurophysiol. 32: $386-401$.

Brugge, J. F., E. Javel, and L. M. Kitzes (1978) Signs of functional maturation of peripheral auditory system in discharge patterns of neurons in anteroventral cochlear nucleus of kitten. J. Neurophysiol. 41: $1557-1579$.

Brugge, J. F., L. M. Kitzes, and E. Javel (1981) Postnatal development of frequency and intensity sensitivity of neurons in the anteroventral cochlear nucleus of kittens. Hear. Res. 5: 217-229.

Carlier, E. M. Abonnenc, and R. Pujol (1975) Maturation des réponses unitaires à la stimulation tonale dans le nerf cochléaire du chaton. J. Physiol. (Paris) 70: 129-138.

Carlier, E., M. Lenoir, and R. Pujol (1979) Development of cochlear frequency selectivity tested by compound action potential tuning curves. Hearing Res. 1: 197-201.

Ferrington, D. G., and M. J. Rowe (1980) Functional capacities of tactile afferent fibres in neonatal kittens. J. Physiol. (Lond.) 307: $335-353$.

Ferrington, D. G., and M. J. Rowe (1982) Specificity of connections and tactile coding capacities in cuneate nucleus of the neonatal kitten. J. Neurophysiol. 47: 622-640.

Gaumond, R. P., C. E. Molnar, and D. O. Kim (1982) Stimulus and recovery dependence of cat cochlear nerve spike fiber discharge probability. J. Neurophysiol. 48: 856-873.

Ginzberg, R. D., and D. K. Morest (1983) A study of cochlear innervation in the young cat with the Golgi method. Hear. Res. 10: 227246.

Goldberg, J. M., and P. B. Brown (1969) Response of binaural neurons of dog superior olivary complex to dichotic tonal stimuli: Some physiological mechanisms of sound localization. J. Neurophysiol. 32: $613-636$.

Greenwood, D. D. (1961) Critical bandwidth and the frequency coordinates of the basilar membrane. J. Acoust. Soc. Am. 33: 1344-1356.

Johnson, D. H. (1980) The relationship between spike rate and synchrony in responses of auditory-nerve fibers to single tones. J. Acoust. Soc. Am. 68: 1115-1122.

Johnson, D. H., and A. Swami (1983) The transmission of signals by auditory-nerve fiber discharge patterns. J. Acoust. Soc. Am. 74: 493501.

Lavine, R. A. (1971) Phase-locking in response of single neurons in cochlear nuclear complex of the cat to low-frequency tonal stimuli. J. Neurophysiol. 34: 467-483.

Liberman, M. C. (1982) The cochlear frequency map for the cat: Labeling auditory-nerve fibers of known characteristic frequency. J. Acoust. Soc. Am. 72: 1441-1449.

Lippe, W., and E. W. Rubel (1983) Development of the place principle: Tonotopic organization. Science 219: 514-516.

Moore, D. R. (1981) Development of the cat peripheral auditory system: Input-output functions of cochlear potentials. Brain Res. 219: 29-44.

Pujol, R., and R. Marty (1970) Postnatal maturation of the cochlea of the cat. J. Comp. Neurol. 139: 115-126.

Pujol, R., E. Carlier, and C. Devigne (1978) Different patterns of cochlear innervation during the development of the kitten. J. Comp. Neurol. 177: 529-536.

Romand, R. (1971) Maturation des potentiels cochléaires dans la période périnatale chez le chat et chez le cobaye. J. Physiol. (Paris) 63: $763-782$.

Romand, R. (1979) Development of auditory nerve activity in kittens Brain Res. 173: 554-556.

Romand, R. (1983) Development of the cochlea. In Development of Auditory and Vestibular Systems, R. Romand, ed., pp. 47-88, Academic Press, Inc., New York.

Romand, R., and M. -R. Romand (1982) Myelination kinetics of spiral ganglion cells in kitten. J. Comp. Neurol. 204: 1-5.

Romand, R., A. Sans, M. -R. Romand, and R. Marty (1976) The structural maturation of the stato-acoustic nerve in the cat. J. Comp. Neurol. 170: 1-16.

Romand, R., M. -R. Romand, C. Mulle, and R. Marty (1980) Early stages of myelination in the spiral ganglion cells of the kitten during development. Acta Otolaryngol. 90: 391-397.

Rose, J. E., N. B. Gross, C. D. Geisler, and J. E. Hind (1966) Some neural mechanisms in the inferior colliculus of the cat which may be relevant to localization of a sound source. J. Neurophysiol. 29: 288314.

Rose, J. E., J. F. Brugge, D. J. Anderson, and J. E. Hind (1967) Phaselocked response to low-frequency tones in single auditory nerve fibers of the squirrel monkey. J. Neurophysiol. 30: 769-793.

Rose, J. E., L. M. Kitzes, M. M. Gibson, and J. E. Hind (1974) Observations on phase-sensitive neurons of anteroventral cochlear nucleus of the cat: Nonlinearity of cochlear output. J. Neurophysiol 37: $218-253$

Rubel, E. W., and B. M. Ryals (1983) Development of the place principle: Acoustic trauma. Science 219: 512-514.

Russell, I. J., and P. M. Sellick (1978) Intracellular studies of hair cells in the mammalian cochlea. J. Physiol. (Lond.) 284: 261-290.

Saunders, J. C., J. A. Kaltenbach, and E. M. Relkin (1983) The structural and functional development of the outer and middle ear. In Development of Auditory and Vestibular Systems, R. Romand, ed. pp. 3-25, Academic Press, Inc., New York.

Sinex, D. G., and C. D. Geisler (1983) Responses of auditory-nerve fibers to consonant-vowel syllables. J. Acoust. Soc. Am. 73: 602-615.

Sobkowicz, H. M., J. E. Rose, G. E. Scott, and S. M. Slapnick (1982) Ribbon synapses in the developing intact and cultured organ of Corti in the mouse. J. Neurosci. 2: 942-957.

Walsh, E. J., J. McGee, and E. Javel (1982) Development of auditory nerve fiber responses in kittens. Soc. Neurosci. Abstr. 8: 345

Wever, E. G. (1949) Theory of Hearing, John Wiley \& Sons, New York. Young, E. D., and M. B. Sachs (1979) Representation of steady-state vowels in the temporal aspects of the discharge patterns of populations of auditory-nerve fibers. J. Acoust. Soc. Am. 66:1381-1403. 